\title{
Voronoi Image Segmentation and Its Applications to Geoinformatics
}

\author{
Ickjai Lee and Kyungmi Lee and Christopher Torpelund-Bruin \\ School of Business, Cairns Campus, Smithfield, Queensland 4870, Australia \\ Email: \{Ickjai.Lee, Joanne.Lee, Christopher.Torpelund\}@jcu.edu.au
}

\begin{abstract}
As various geospatial images are available for analysis, there is a strong need for an intelligent geospatial image processing method. Segmenting and districting digital images is a core process and is of great importance in many geo-related applications. We propose a flexible image segmentation framework based on generalized Voronoi diagrams through Euclidean distance transforms. We introduce a three-scan algorithm that segments images in $O(N)$ time when $N$ is the number of pixels. The algorithm is capable of handling generators of complex types (point, line and area), Minkowski metrics and different weights. This paper also provides applications of the proposed method in various geoinformation datasets. Illustrated examples demonstrate the usefulness and robustness of our proposed method.
\end{abstract}

Index Terms-Image segmentation, Voronoi diagram, Geoinformatics, Raster

\section{INTRODUCTION}

Districting is to partition a study region $S$ into meaningful and manageable smaller units called districts or zones. It is one of the widely used methods in GIS (Geographic Information Systems) that allows the user to define and manipulate aggregations of geospatial data. Basically, it segments $S$ into mutually exclusive and collectively exhaustive regions of influence. Examples of districting include political boundaries, census tracts, catchment areas, segmented market areas, delivery areas and sales territories. Districting not only produces a geometric tessellation, but it provides a robust framework for various topological questions such as nearest neighbor questions, adjacent neighbor queries and geospatial lag questions. Mostly, it has been studied with the vector data format since most GIS are basically built on the vector data format for geospatial analysis. Due to the availability of areal photography, wide-spread Google maps, and remotely sensed images, the need for geospatial image districting is in great demand to handle these easily accessible geospatial images.

The Voronoi Diagram (VD) has long been a popular approach in vector districting [1]. It can be obtained by assigning every point in the study region to the nearest target generator in a certain distance metric. Resulting Voronoi vertices, edges and regions serve as a basis for points, lines and areas of the vector format. In this vector districting approach, typically generators are limited to points, the underlying metric is replaced by the Euclidean metric and weights of generators are assumed to be invariant. Some vector districting based on generalized
VDs have been proposed in order to overcome these limitations [1]. Despite of the wide use of the VD in vector districting, its use in geospatial image districting has attracted less attention [2].

This paper introduces a flexible geospatial image districting framework based on generalized VDs. It proposes a three-scan algorithm that segments geospatial images in $O(N)$ time when $N$ is the number of pixels. The algorithm is capable of handling generators of complex types (point, line and area), Minkowski metrics and different weights. This paper also provides applications of the proposed method to various geoinformation datasets.

The rest of this paper is organized as follows. Section II briefly overviews the VD and its generalizations to complex primitives, Minkowski metrics and different weights. Section III outlines details of our proposed three-scan algorithm whilst Section IV provides applications with images from Google Earth (http://earth.google.com/) and GeoWeb 2.0. Section V draws conclusive remarks and suggests future work.

\section{VORONOI DIAGRAM SEGMENTATION}

The Voronoi diagram models natural territories of events [3]. It has been widely used in geoinformatics [1], [4-6]. It captures estimates of the service areas, and models impact areas of a certain target.

Let $P=\left\{p_{1}, p_{2}, \ldots, p_{\mathrm{k}}\right\}$ be a set of generators. For any point $x$ in the plane, $d\left(x, p_{\mathrm{i}}\right)$ denotes the distance from point $x$ to a generator point $p_{\mathrm{i}}$. Every location $x$ in $S$ can be assigned to the closest generator $p_{\mathrm{i}} \in P$ with a certain distance metric $d$. If it is equally close to two generators in $P$ then the location becomes a part of Voronoi edge while if it is equally close to more than two generators then the location becomes a Voronoi vertex. This assignment results in a mutually exclusive and collectively exhaustive tessellation called Voronoi diagram. The resulting dominance region of $p_{\mathrm{i}}$ over $p_{\mathrm{j}}$ can be defined as:

$$
\operatorname{dom}\left(p_{i}, p_{j}\right)=\left\{p \mid d\left(p, p_{i}\right) \leq d\left(p, p_{j}\right)\right\} .
$$

For the generator point $p_{\mathrm{i}}$, the Voronoi region of $p_{\mathrm{i}}$ can be defined by:

$$
V\left(p_{i}\right)=\bigcap_{j \neq i} \operatorname{dom}\left(p_{i}, p_{j}\right) .
$$

The partition of the plane into subsequent Voronoi regions $V\left(p_{1}\right), V\left(p_{2}\right), \ldots, V\left(p_{\mathrm{k}}\right)$ is called the generalized Voronoi diagram. The bounding regions of $V\left(p_{\mathrm{i}}\right)$ are known Voronoi boundaries and depending on the primitive used as the generator, such as points, lines or 


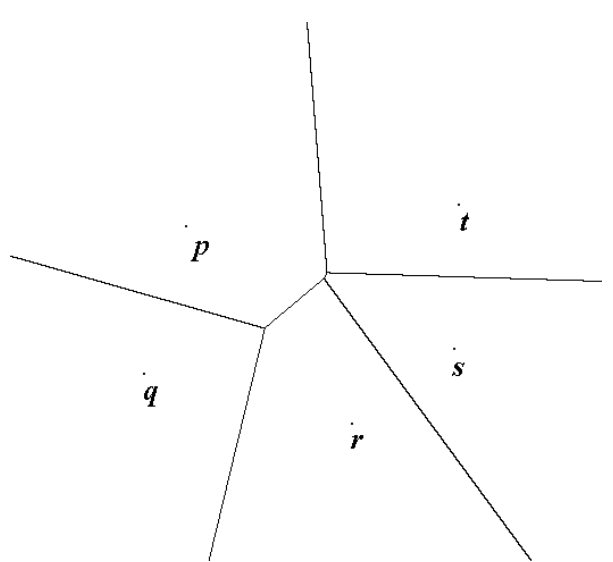

Figure 1. The ordinary VD or $P=\{p, q, r, s, t\}$.

polygons and the metric space used, will result in a series of polygons and arcs made up of lines and Bezier curves [1]. This study considers three different types of generalizations: Minkowski metrics, weights and complex primitives. Fig. 1 depicts the ordinary VD of 5 generators.

\section{A. Minkowski Voronoi Diagrams}

The most popular distance metric $d$ is the Euclidean distance. It is an instance of the Minkowski metric and in

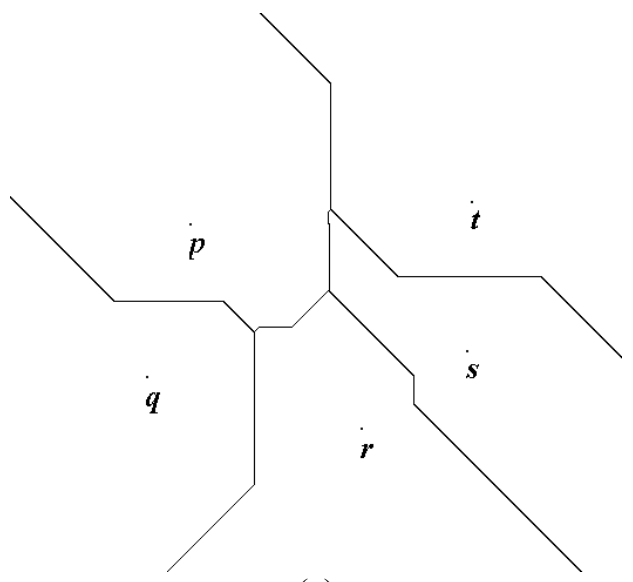

(a)

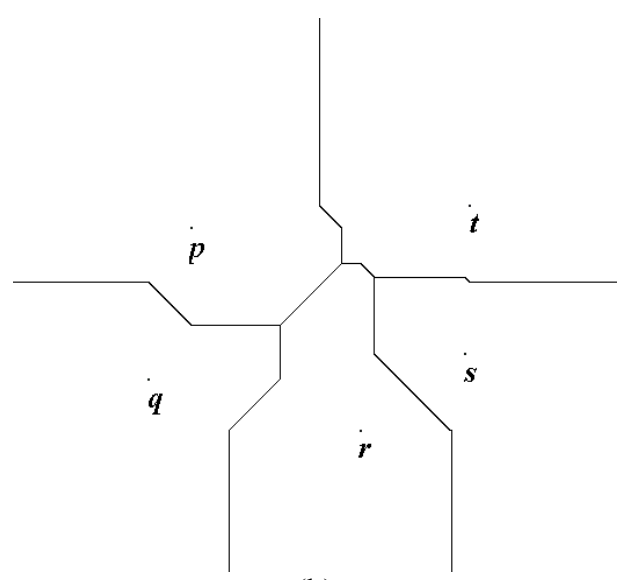

(b)

Figure 2. Minkowski VDs: (a) Manhattan VD of $P$; (b) Maximum VD of $P(P$ is the same as in Fig. 1).

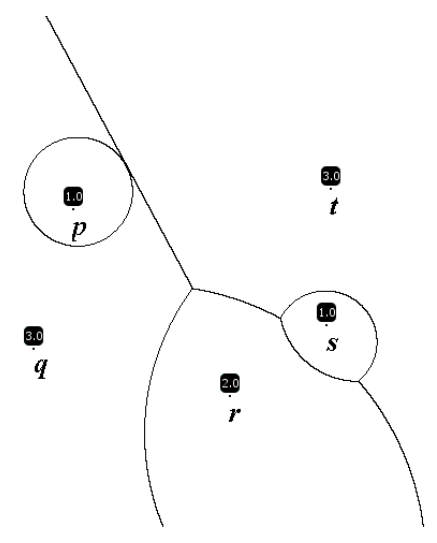

(a)

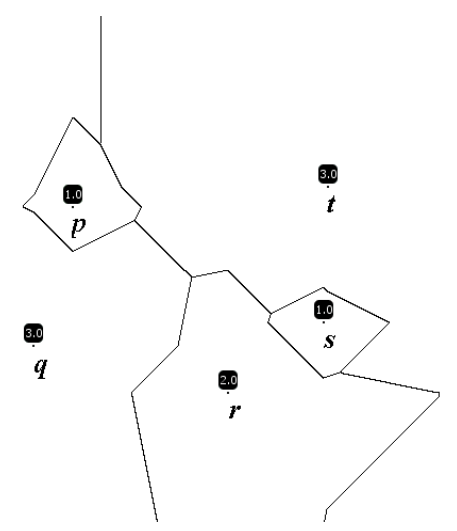

(b)

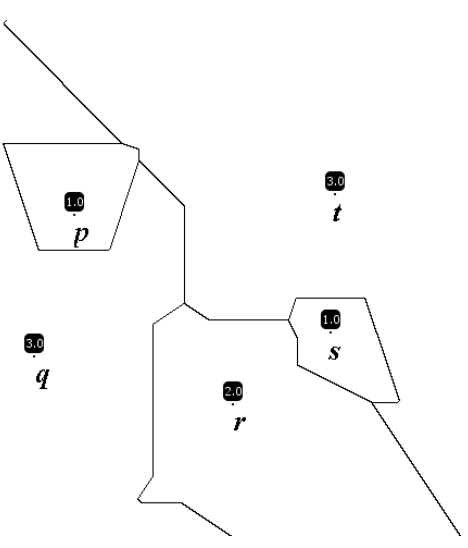

(c)

Figure 3. Weighted VDs: (a) Weighted Euclidean VD of $P$; (b) Weighted Manhattan VD of $P$; (c) Weighted maximum VD of $P(P$ is the same as in Fig. 1).

urban geography another instance of the Minkowski metric, the Manhattan distance, better approximates real world situations [7]. Thus, in this study we utilize three instances of the Minkowski metric, namely $p=1, p=2$ and $p=\infty$. Let $d_{L p}: \mathfrak{R}^{2} \times \mathfrak{R}^{2} \rightarrow \mathfrak{R}$ denote the Minkowski metric defined by

$d_{L p}(r, s)=\left(\sum\left|r_{i}-s_{i}\right|^{p}\right)^{1 / p}(p \in \mathfrak{R} \cup\{\infty\}, p \geq 1)$. 
If $p=1$, then $d_{L l}(r, s)=\left|r_{x}-s_{x}\right|+\left|r_{y}-s_{y}\right|$ is the Manhattan metric. The Minkowski metric becomes the Euclidean metric when $p=2$. If $p=\infty$, then the Minkowski metric becomes $d_{L \infty}(r, s)=\max \left\{\left|r_{x}-s_{x}\right|, \mid r_{y}\right.$ $\left.s_{y} \mid\right\}$, which is called the maximum metric. Fig. 2 depicts corresponding Manhattan VD of $P$ and maximum VD of $P$ where $P$ is the same as in Fig. 1. It is obvious to note that different metrics result in different tessellations.

B. Weighted Voronoi Diagrams

There exist a variety of functional forms for calculating the weighted distance and many weighted VDs are possible. In this study, the multiplicatively weighted distance is used to model different weights in the Minkowski metric. It is defined as follows:

$$
d_{m w}\left(p, p_{i}\right)=\frac{1}{w_{i}}\left|p-p_{i}\right|\left(w_{i}>0\right) .
$$

Fig. 3 depicts several different weighted VDs of $P$.

\section{Voronoi Diagrams with Complex Primitives}

The real world is obviously non-trivial and it is the goal of GIS to represent real world objects (roads, land use, elevation) and associated attributes for capturing, storing, analyzing and managing data which are spatially referenced to the Earth. However, a browse through recent literature on GIS and VD reveals that most represent real world objects of interest as point sets, and

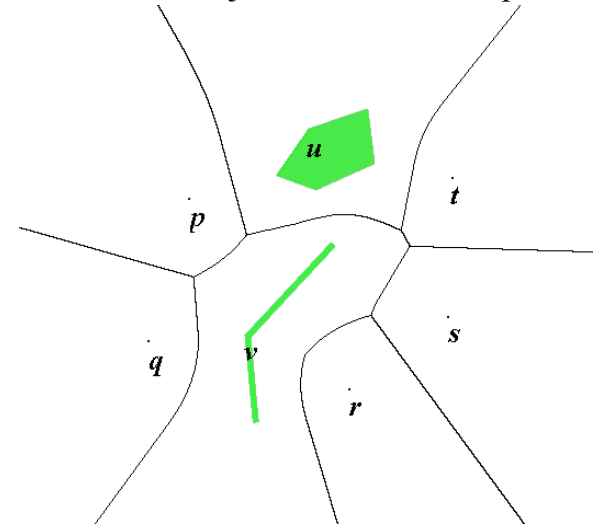

(a)

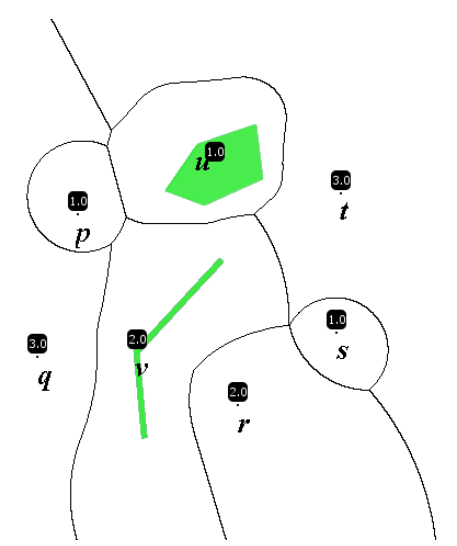

(b)

Figure 4. Generalized VDs with complex data types: (a) Euclidean VD of $P^{\prime}$; (b) Weighted Manhattan VD of $P^{\prime}$; (c) Weighted maximum VD of $P^{\prime}\left(P^{\prime}=P+\{(u, 1),(v, 2)\}\right.$ and $P$ is the same as in Fig. 1).

most methods of generating VDs are all vector-based.
Line and area features can be used to represent objects where the geometric space can not be ideally represented by point sets. But as with the case for weighted VDs, vector-based methods for generating VDs work well for point datasets, but are not efficient for line segments, area features [2], [8].

The distance from a point $x$ to a complex primitive $p_{i}$ is defined as:

$$
d\left(x, p_{i}\right)=\min x_{i}\left\{\left|x-x_{i}\right| \mid x_{i} \in p_{i}\right\} .
$$

Fig. 4 shows generalized VDs with complex data types. Fig. 4(a) depicts the Euclidean VD of $\mathrm{P}^{\prime}$ while Fig. 4(b) illustrates the weighted $\mathrm{VD}$ of $\mathrm{P}^{\prime}$ where $\mathrm{P}^{\prime}=\mathrm{P}+$ $\{(\mathrm{u}, 1),(\mathrm{v}, 2)\}$ and $\mathrm{P}$ is the same as in Fig. 1. The line segment $\mathrm{v}$ has a weight 2 associated and the areal generator $\mathrm{u}$ has 1 associated. Fig. 5 depicts the generalized Manhattan VD of P'.

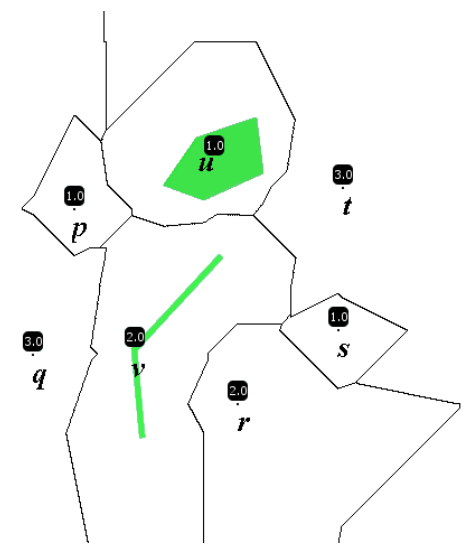

Figure 5. Generalized Manhattan VD of $P^{\prime}\left(P^{\prime}\right.$ is the same as in Fig. 4).

\section{RASTER VORONOI DIAGRAMS THROUGH EUCLIDEAN DISTANCE TRANSFORMS}

The basic idea of raster VD is to expand the VD incrementally by adding one pixel at a time for all pixels in the image by considering the number of neighbors or direction of connection [9]. The raster VD is represented by a discrete grid lattice of size $M \times M$ which gives $M^{2}$ generators in the plane. The development of the raster VD is based heavily on the advances in distance transformations. Distance transforms are characterized by the mappings of each image pixel (foreground) into its smallest distance to regions of interest (background) [10]. Recently, efficient and precise Euclidean Distance Transforms (EDTs) emerged as a result of the difficulties in processing the non-local properties of the Euclidean metric on a discrete grid lattice which becomes a problem when trying to find the bisecting pixels between generators[11]-[13].

\section{A. Euclidean Distance Transform}

EDTs can be divided into mainly two categories based on the order used to scan the pixels [11]: iterative scan algorithms and sequential algorithms. Iterative algorithms use wave scans over points in the background image which are seen as sources from which distance values are 
calculated for the foreground image. The waves propagate out from the sources like how a grassfire would burn from the source of ignition outwards. These algorithms are suited to parallel processing computer systems and time complexity for such parallel processing is typically $O(M)$ for an image of $M \times M$. The same propagation wave scan is possible with a single processor with a time complexity of $O\left(M^{2}\right)$ [10]. Therefore most iterative algorithms are inefficient as many pixels are often unnecessarily updated. Sequential algorithms are characterized by the number of neighbors that are considered and the number of sweeps from left to right and from top to bottom, and reverse sweeps from right to left and from bottom to the top of the image. The number of neighbors ranges from 4-neighborhood all the way to arbitrarily large sizes. The shape of neighbors being considered also differs between algorithms and there is a trade-off between the size and shape of neighborhood with the number of iterations needed to achieve a solution and the quality of approximation [14]. Even if sequential scan algorithms are more efficient than iterative algorithms, many pixels still need to be processed more than once in sequential algorithms. This becomes more of a problem for non-convex domains which usually require multiple passes before achieving the final distance values [12].

Recently, fast sequential scan algorithms requiring $O(N)$ time have been proposed [15], [16] where $N$ is the number of generators. Cuisenaire and Macq [15] proposed a fast EDT by propagation through multiple neighborhoods and bucket sorting. However, complex multiple neighborhoods and bucket sorting are considerable overheads of their approach. This was further improved by Shih and $\mathrm{Wu}$ [16] who employed a 3 $\times 3$ neighborhood instead of complex neighborhoods. They computed the exact EDT through the two scan algorithm using the simpler $3 \times 3$ neighborhood. However, their approach is limited to simple primitives (point type) and unweighted primitives. Therefore, it is not straightforward to compute generalized weighted raster VDs of complex primitives. We further develop Shih and $\mathrm{Wu}[16]$ 's method and propose a three scan algorithm to deal with multiple metrics, weighted primitives, and complex primitives.

\section{B. Proposed Three-scan Method}

The weighted EDT is processed by propagating the weight values of the primitives and considering the direction of connection for the convex domain. The algorithm requires just three scans over the geospatial image, forward and backwards followed by a forward scan. Distance values are also propagated as squared Euclidean values, skipping the square-root calculation as in [16].

Let us consider a background binary image of features $O$ and a foreground image of DTs over the background image $F$. Let $q_{1}, q_{2}, \ldots, q_{8}$ be the eight neighbors of the shaded cell $p$ as seen in Fig. 6(a). In the Manhattan metric, the mask $q_{1}, q_{3}$ is defined by $N_{1}(p)$ and $q_{5}, q_{7}$ is defined by $N_{2}(p)$ which can be seen in Fig. 6(b). In the

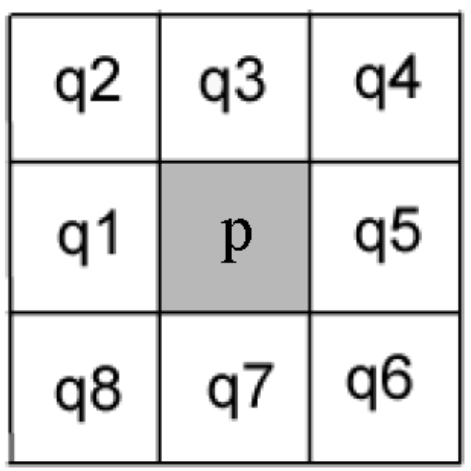

(a)

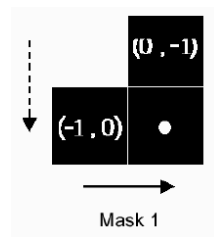

(b)
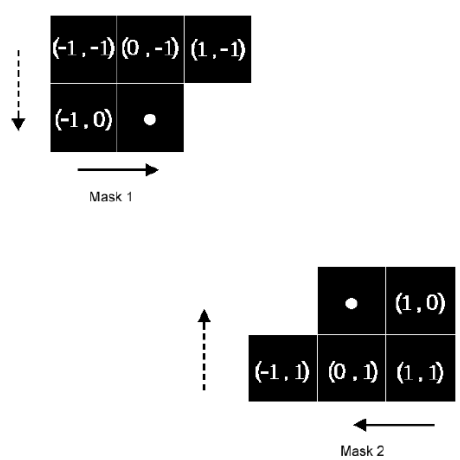

(c)

Figure 6. Neighbors and scan masks: (a) The eight neighbors of $p$; (b) The Manhattan neighbor mask; (c) The chessboard and Euclidean neighbor mask.

Euclidean and maximum metrics, the mask $q_{1}, q_{2}, q_{3}, q_{4}$ is defined by $N_{1}(p)$ and $q_{5}, q_{6}, q_{7}, q_{8}$ is defined by $N_{2}(p)$ which can be seen in Fig. 6(c). The mask $N_{1}(p)$ is used for forward scan whilst the mask $N_{2}(p)$ is used for backward scan. At each point $p$ the minimum distance value is propagated by the neighbors in the masks. The algorithm is given by:

$$
\begin{gathered}
X^{\prime}(p)=\min _{q \in N_{1}} X^{\prime}(q)+1, \\
X^{\prime \prime}(p)=\min _{q \in N_{2}}\left[X^{\prime}(p), X^{\prime \prime}(q)+1\right],
\end{gathered}
$$

where $X^{\prime}(p)$ is computed in a left to right, top to bottom scan and $X^{\prime \prime}(p)$ is computed in a reverse scan.

Let $R(p)$ be the relative vector coordinates of pixel $p$, which records the horizontal and vertical pixel-distances between $p$ and the closest background pixel. Let $h(p, q)$ be the difference of the squared Euclidean distance between $p$ and $q \in N_{1} \cup N_{2}$. Let $G(p, q)$ be the 
difference of the vector distance used to calculate the distance values of the pixels preceding $p$ given by the masks. The $w_{q}$ value represents the significance (weight) from the background pixel. The $h(p, q)$ and $G(p, q)$ can be computed in the following way:

$$
\begin{aligned}
& h(p, q)=\left\{\begin{array}{c}
2 R_{x}(q)+1 \\
\text { if } q \in\left\{q_{1}, q_{5}\right\}, \\
2 R_{y}(q)+1 \\
\text { if } q \in\left\{q_{3}, q_{7}\right\}, \\
2 R_{x}(q)+R_{y}(q)+1 \\
\text { if } q \in\left\{q_{2}, q_{4}, q_{6}, q_{8}\right\} .
\end{array}\right\} \\
& G(p, q)=\left\{\begin{array}{cc}
(1,0) & \text { if } q \in q_{1} \\
(1,-1) & \text { if } q \in q_{2} \\
(0,-1) & \text { if } q \in q_{3} \\
(-1,-1) & \text { if } q \in q_{4} \\
(-1,0) & \text { if } q \in q_{5} \\
(-1,1) & \text { if } q \in q_{6} \\
(0,1) & \text { if } q \in q_{7} \\
(1,1) & \text { if } q \in q_{8}
\end{array}\right\}
\end{aligned}
$$

\section{Algorithms and Analysis}

a) Process generators to Cartesian coordinates

b) (Forward Scan) For each pixel $p \in F$

i) set all $\mathrm{p} \in \mathrm{F}=\infty$

ii) for each neighborhood $q \in N_{1}(p)$

- $f_{\text {new }}(p)=\left(R_{x}(q)^{2}+R_{y}(q)^{2}+h(p, q)\right) / w_{q}$

- $f(p)=\min \left(f(p), f_{\text {new }}(p)\right)$

iii) modify: $R(p)=R(q)+G(p, q)$

c) (Reverse Scan) For each pixel $p \in F$

i) for each neighborhood $q \in N_{2}(p)$

- $f_{\text {new }}(p)=\left(R_{x}(q)^{2}+R_{y}(q)^{2}+h(p, q)\right) / w_{q}$

- $f(p)=\min \left(f(p), f_{\text {new }}(p)\right)$

ii) modify: $R(p)=R(q)+G(p, q)$

d) Repeat Step b) Forward Scan

e) Scan along top left corner until highest squared Euclidean distance bisection found for each generator $g \in G$;

1) record bisecting line between regions with highest squared Euclidean distance;

2) recursively follow intersections of 3 or more generators and record bisecting; line between regions

3) if intersection already recorded then end current iteration;

4) if back to first region and completed then end;

The system starts with Step a) by initializing all the primitives into their Cartesian coordinates. This deals

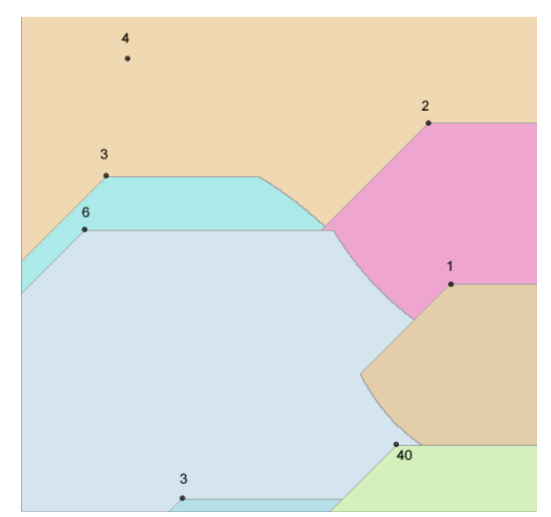

(a)

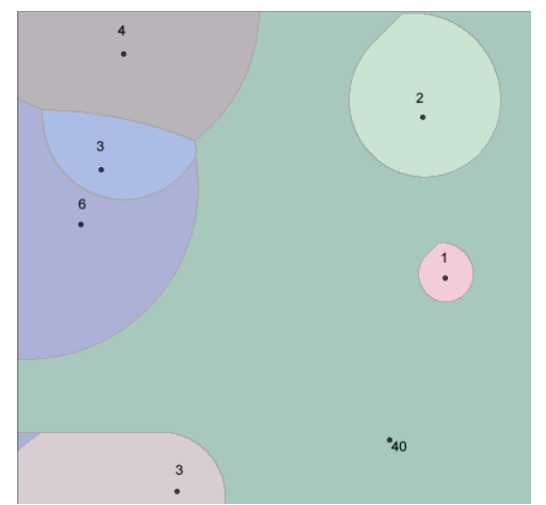

(b)

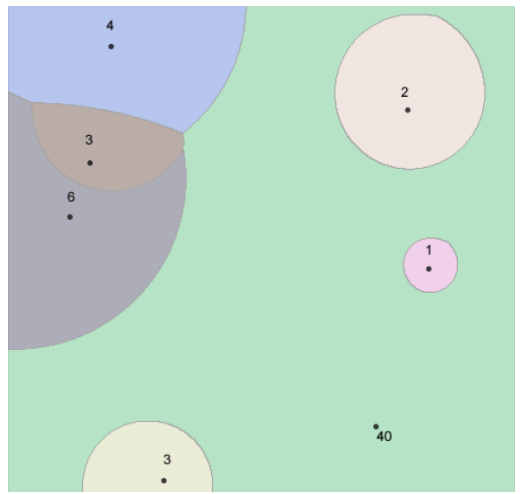

(c)

Figure 7. The individual scans to achieve weighted VDs: (a) First scan; (b) Second scan; (c) Third scan.

with complex primitives like for lines and area generators. Step b) (Fig. 7(a)) details the forward scan propagation of using $N_{1}(p)$. Firstly all pixels $p$ need to be initialized with a non-processed value. Next the pixels $q$ in the mask around $p$ is processed to determine the closest background pixel. Next the relative distance from the background object is found either being forward or backwards, and is updated to $R(p)$. Step c) (Fig. 7(b)) details the same process in the reverse scan using $N_{2}(p)$. In order to deal with the convex domain caused by using weighted primitives a third raster scan with $N_{1}(p)$ is needed as seen by Step d) (Fig. 7(c)). After the first 4 steps, the system has finished the DT and constructed $F$. From this, Step e) details the vectorization of the raster image $F$ to construct a graph of the dominance region boundaries used for analysis and producing results to various topological query types. The process recursively 
follows the highest squared Euclidean distance values in $F$ which define the bounding regions until 3 or more intersections of generators are found at which time the process runs again from the first bounding region on the left. The process continues until all regions have been processed by recursion. These steps are visualized in Fig. 8.

\begin{tabular}{l|l|l|l|l|l}
1.0 & 0.5 & 1.0 & 2.5 & 5.0 & 4.0 \\
\hline 0.5 & 0.0 & 0.5 & 2.0 & 2.0 & 1.0 \\
\hline 1.0 & 0.5 & 1.0 & 2.5 & 1.0 & 0.0 \\
\hline 2.5 & 2.0 & 2.0 & 1.0 & 2.0 & 1.0 \\
\hline 5.0 & 4.0 & 1.0 & 0.0 & 1.0 & 4.0 \\
\hline 8.5 & 5.0 & 2.0 & 1.0 & 2.0 & 5.0
\end{tabular}

(a)

\begin{tabular}{l|l|l|l|l|l}
1.0 & 0.5 & 1.0 & 2.5 & 5.0 & 4.0 \\
\hline 0.5 & 0.0 & 0.5 & 2.0 & 2.0 & 1.0 \\
\hline 1.0 & 0.5 & 1.0 & 2.5 & 1.0 & 0.0 \\
\hline 2.5 & 2.0 & 2.0 & 1.0 & 2.0 & 1.0 \\
\hline 5.0 & 4.0 & 1.0 & 0.0 & 1.0 & 4.0 \\
\hline 8.5 & 5.0 & 2.0 & 1.0 & 2.0 & 5.0
\end{tabular}

(b)

\begin{tabular}{l|l|l|l|l|l}
1.0 & 0.5 & 1.0 & 2.5 & 5.0 & 4.0 \\
\hline 0.5 & 0.0 & 0.5 & 2.0 & 2.0 & 1.0 \\
\hline 1.0 & 0.5 & 1.0 & 2.5 & 1.0 & 0.0 \\
\hline 2.5 & 2.0 & 2.0 & 1.0 & 2.0 & 1.0 \\
\hline 5.0 & 4.0 & 1.0 & 0.0 & 1.0 & 4.0 \\
\hline 8.5 & 5.0 & 2.0 & 1.0 & 2.0 & 5.0
\end{tabular}

(c)

\begin{tabular}{|l|l|l|l|l|l|}
1.0 & 0.5 & 1.0 & 2.5 & 5.0 & 4.0 \\
\hline 0.5 & 0.0 & 0.5 & 2.0 & 2.0 & 1.0 \\
\hline 1.0 & 0.5 & 1.0 & 2.5 & 1.0 & 0.0 \\
\hline 2.5 & 2.0 & 2.0 & 1.0 & 2.0 & 1.0 \\
\hline 5.0 & 4.0 & 1.0 & 0.0 & 1.0 & 4.0 \\
\hline 8.5 & 5.0 & 2.0 & 1.0 & 2.0 & 5.0
\end{tabular}

(d)

Figure 8. Vectorization of the geospatial image with (1), (2), (3) and (4) referring to the steps of the algorithm at Step e).

\section{APPLICATIONS TO GEOINFORMATICS}

\section{A. Market Analysis}

Market analysis is an investigation of a market that is used to inform a firm's planning activities particularly around decisions of: inventory, purchase, work force expansion/contraction, facility expansion, purchases of capital equipment, promotional activities, and many other aspects of a company. An important part of market analysis is the discovery of new strategies that can allow an organization to concentrate its limited resources on the greatest opportunities to increase sales and achieve a sustainable competitive advantage. Thus, segmenting an interested target study region into meaningful and catchable districts is of great importance in market analysis.

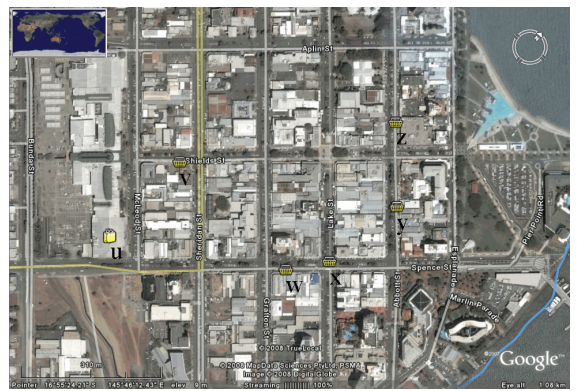

(a)

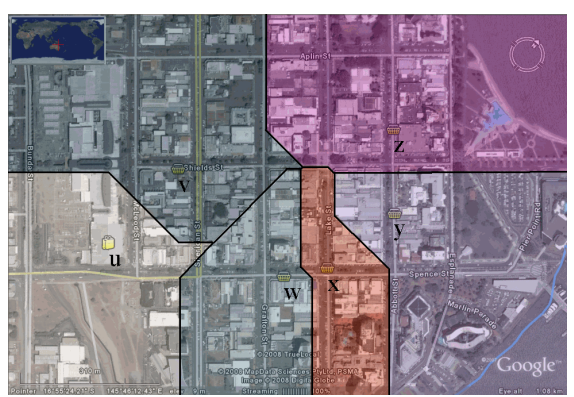

(b)

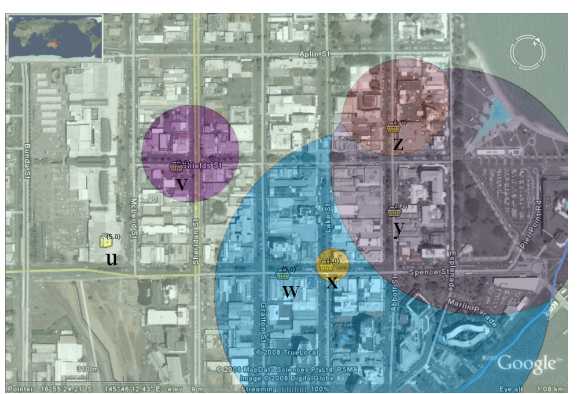

(c)

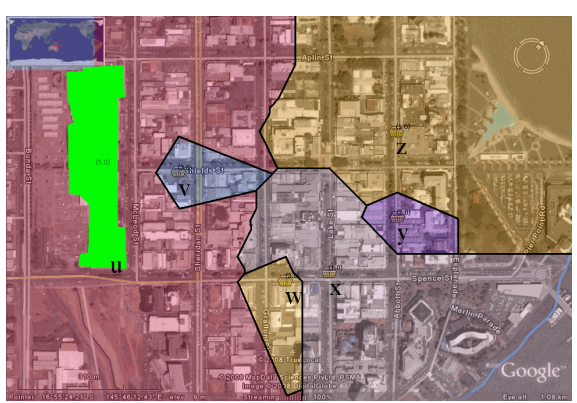

(d)

Figure 9. Geospatial image districting: (a) Urban suburbs of Cairns with 6 shopping centers: (b) Ordinary VD; (c) Weighted VD; (d) Weighted VD in $d_{L 1}$ with complex primitives.

Fig. 9 shows a series of market districting of realworld images with our proposed three-scan algorithm. Fig. 9(a) depicts a snapshot of Google Earth around urban areas of Cairns in Australia with 6 shopping centers $\{(u, 5),(v, 3),(w, 2),(x, 1),(y, 2),(z, 1)\}$. Shopping center $u$ 
is a big shopping complex forming an areal shape with a higher weigh 5. Fig. 9(b) shows the ordinary VD in the Manhattan metric. Note that, the Manhattan metric better approximates real world situations in urban geography [7]. Therefore, Fig. 9(b) shows an informative urban districting. Fig. 9(c) illustrates the same study region with weights considered. It provides a more meaning districting when weights (sizes or the number of shops) of shopping centers are considered. Fig. 9(d) models when the areal unit of the shopping center $u$ is considered along with weights in the Manhattan metric.

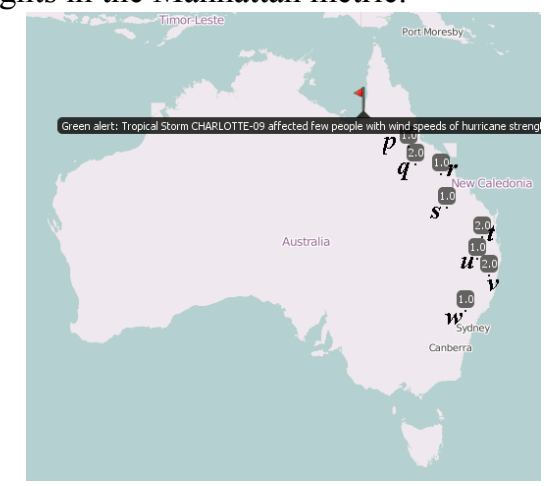

(a)

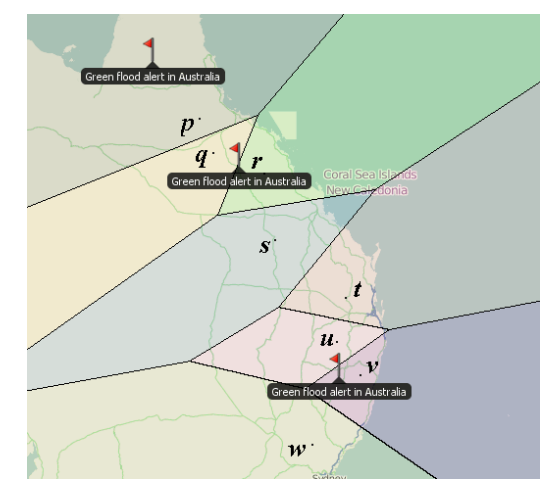

(b)

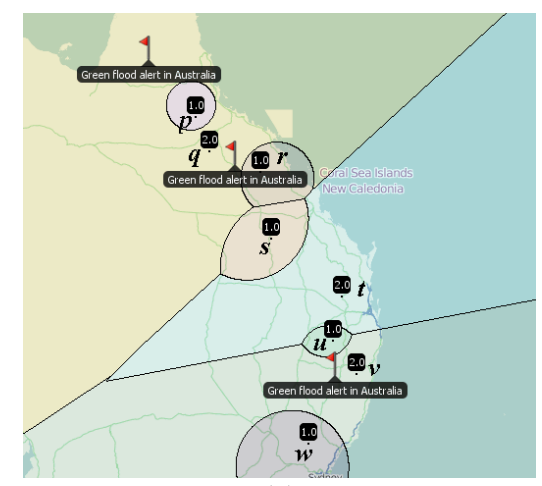

(c)

Figure 10. Emergency management: (a) A tropical storm Charlotte and 8 disaster management units $D=\{(p, 1),(q, 2),(r, 1),(s, 1),(t, 2),(u, 1)$, $(v, 2),(w, 1)\} ;$ (b) Ordinary VD of $D$; (c) Weighted VD of $D$. of human civilization and settlement, people have been faced with a myriad of risks and dangers, effecting both individuals and communities. However, the Earth seems to be undergoing a notable change that has the potential, if no corrective action is taken, to lead to the greatest disasters that human civilization has ever experienced. More than four times the number of natural disasters are occurring now than just two decades ago. The world suffered about 120 natural disasters per year in the early 1980s. That figure now stands at about 500 per year and is predicted only to increase more. Researchers say that rising green house gas emissions are the major cause of global warming.

In many real world situations, disasters have many various components like different sizes, speed and spreading, impacts on neighboring regions and time specific values. A small bush fire should not be equally thought of to be as dangerous as burning homes and buildings. In the same regard, emergency management units are widely varied and there are many reasons why they should not be equally weighted. For example a regional volunteer fire department should not be thought to provide the same abilities as that of a city fire department which have more fire-engines and firefighters available. Also, the distance from one point to the next should not be taken for granted to be in Euclidean distance (A direct route from A to B). The streets within a city are rarely so efficient and require many turns and other factors to be considered. VDs are capable of modeling all of these scenarios and more.

Fig. 10 illustrates an example of emergency management with our framework. Fig. 10(a) depicts a current real-world disaster extracted from GeoWeb 2.0 on 19th January 2009. There is one reported disaster, tropical storm called Charlotte. We have temporarily placed 8 emergency management units with different weights (higher weights with bigger units) to protect local areas and handle damages from the tropical storm. Due to this storm, there reported three floods in nearby areas. Fig. 10(b) shows these floods extracted from GeoWeb 2.0 with the ordinary VD of the temporal emergency units. This is of particular interest when emergency units are of the same size and floods equally spread to every direction. This diagram clearly shows that emergency unit $p$ is the closest unit to the flood in the northern side, $r$ is to the one in the middle, and $v$ is to the one in the south. However, this scenario is better modeled by weighted VDs. Fig. 10(c) depicts a new tessellation with weights considered. Now, a big emergency unit $q$ is in charge of the two disasters in the northern side whilst $v$ is in the one in the south. Different uses of VDs generate different tessellations. Our framework provides a set of different tessellations from which a user can select one that best suits the case at hand.

\section{B. Emergency Management}

A disaster is the impact of a natural or man-made hazard that negatively affects society or the environment. There is nothing new about disasters. Since the beginning

\section{SUMMARY AND FUTURE WORK}


In this paper, we propose a general-purpose geospatial image segmenting model through generalized raster VDs using EDTs. We describe the generalizations of raster VDs and distance transforms to complex primitives, weighted primitives and Minkowski metrics. We also provide a set of real world examples from Google Earth images to illustrate the usefulness and robustness of our framework. Our proposed algorithm needs $O(N)$ time and supports various districting questions for what-if analysis. A future implementation of our project could explore iterative scan algorithms to see if there is some advantages to this method. Also, more generalizations of the VD like power and the network VD could be researched to include the elements of time, networks and underlying population information into the analysis of emergency management decision support systems.

\section{REFERENCES}

[1] A. Okabe, B. N. Boots, K. Sugihara, and S. N. Chiu, Spatial Tessellations: Concepts and Applications of Voronoi Diagrams, 2nd ed. West Sussex: John Wiley \& Sons, 2000.

[2] C. Li, J. Chen, and Z. Li, "A Raster-based Method for Computing Voronoi Diagrams of Spatial Objects Using Dynamic Distance Transformation," International Journal of Geographical Information Science, vol. 13, no. 3, pp. 209-225, 1999.

[3] C. M. Gold, "Problems with Handling Spatial Data - The Voronoi Approach," Canadian Institute of Surveying and Mapping Journal, vol. 45, no. 1, pp. 65-80, 1991.

[4] M. Gahegan and I. Lee, "Data Structures and Algorithms to Support Interactive Spatial Analysis Using Dynamic Voronoi Diagrams," Computers, Environments and Urban Systems, vol. 24, no. 6, pp. 509-537, 2000.

[5] I. Lee and M. Gahegan, "Interactive Analysis using Voronoi Diagrams: Algorithms to Support Dynamic Update from a Generic Triangle-Based Data Structure," Transactions in GIS, vol. 6, no. 2, pp. 89-114, 2002.

[6] I. Lee, R. Pershouse, P. Phillips, and C. Christensen, "What-if Emergency Management System: A Generalized Voronoi Diagram Approach," in Proceedings of the First Pacific-Asia Workshop on Intelligence and Security Informatics, Lecture Notes in Computer Science 4430, C. C. Yang, D. Zeng, M. Chau, K. Chang, Q. Yang, X. Cheng, J. Wang, F.-Y. Wang, and H. Chen, Eds. Chengdu, China: Springer, 2007, pp. 58-69.

[7] E. F. Krause, Taxicab Geometry. California: AddisonWesley, 1975.

[8] L. G. Canton, Emergency Management: Concepts and Strategies for Effective Programs. New Jersey: Wiley, 2007.

[9] C. Li, J. Chen, and Z. Li, "A Raster-based Method for Computing Voronoi Diagrams of Spatial Objects using Dynamic Distance Transformation," International Journal of Geographical Information Science, vol. 13, no. 3, pp. 209-225, 1999.

[10] A. Rosenfeld and J. L. Pfaltz, "Sequential Operations in Digital Picture Processing," Journal of $A C M$, vol. 13, no. 4, pp. 471-494, 1966.

[11] O. Cuisenaire, "Distance Transformations: Fast Algorithms and Applications to Medical Image Processing," Ph.D. dissertation, Universite Catholique de Louvain, Belgique, 1999.

[12] R. Fabbri, L. D. F. Costa, J. C. Torelli, and O. M. Bruno, "2D Euclidean Distance Transform Algorithms: A
Comparative Survey," ACM Computing Surveys, vol. 40, no. 1, pp. 1-44, 2008.

[13] Y. Ge and J. M. Fitzpatrick, "On the Generation ofSkeletons from Discrete Euclidean Distance Maps," IEEE Transactions on Pattern Analysis and Machine Intelligence, vol. 18, no. 11, pp. 1055-1066, 1996.

[14] H. Breu, J. Gil, D. Kirkpatrick, and M. Werman, "Linear Time Euclidean Distance Transform Algorithms," IEEE Transactions on Pattern Analysis and Machine Intelligence, vol. 17, no. 5, pp. 529-533, 1995.

[15] O. Cuisenaire and B. Macq, "Fast Euclidean Distance Transformation by Propagation Using Multiple Neighborhoods," Computer Vision and Image Understanding, vol. 76, no. 2, pp. 163-172, 1999.

[16] F. Y. Shih and Y.-T. Wu, "Fast Euclidean Distance Transformation in Two Scans Using a $3 \quad £ \quad 3$ Neighborhood," Computer Vision and Image Understanding, vol. 93, no. 2, pp. 195-205, 2004.

Ickjai Lee obtained his Ph.D. in School of Electrical Engineering and computer Science from the University of Newcastle, Australia in 2002, his Honours degree in Spatial Science and graduate diploma in Computing from Curtin University of Technology, Australia, and his BSc in Department of Computer Science from the Pusan National University, Korea in 1993.

After his Ph.D., he worked as a postdoctoral research fellow at Newcastle Business School in the University of Newcastle in the areas of artificial intelligence, robot soccer and knowledge representation. Currently, he is a senior lecturer at School of Business in James Cook University, Australia. He has published around 60 articles in refereed journals and conferences. His research areas include geoinformatics, spatial data mining, Voronoi diagram, mobile-intelligence and artificial intelligence.

Dr. Lee is a lifetime member of ACM since 2008 and a member of IEEE since 2004. He is a member of editorial review board of Journal of Computer Information Systems, and is a member of program committee of several conferences. He has regularly reviewed articles for several journals from geoinformatics and information system areas.

Kyungmi Lee received her Ph.D. in School of Computing and Information Technology from Griffith University, Australia in 2007, her Graduate Certificate from School of Electrical Engineering and Computer Science from the University of Newcastle, Australia in 2001, and her BSc in Computer Science from the Pusan National University, Korea in 1993.

She joined Charles Stuart University after her Ph.D. as lecturer. Recently, she joined School of Business in James Cook University as a lecturer. She has been actively involved in collaborative research projects in the areas of machine learning, non-destructive signal processing, geoinformatics and business intelligence.

Dr. Lee has been involved in some academic activities including reviewing articles for journals and conferences, serving as a member of program committee for machine learning related conferences, and supervising students.

Christopher Torpelund-Bruin is currently a Ph.D. candidate at School of Business in James Cook University. He received his BSc with Honours (First class) in School of Math, Physics \& IT in James Cook University, Australia in 2008. His current research areas include geospatial data mining, Web 2.0, and Voronoi diagrams. 